\title{
Using UV light for adhesive remnant removal after debonding of orthodontic accessories
}

Edmilson Nobumito KANESHIMA(a) Sandrine Bittencourt BERGER(b) Thais Maria Freire FERNANDES(a) Maria Fidela de Lima NAVARRO(c) Paula Vanessa Pedron OLTRAMARI ${ }^{(a)}$

(a) Universidade do Norte do Paraná - Unopar, Department of Orthodontics, Londrina, PR, Brazil.

(b) Universidade do Norte do Paraná - Unopar, Department of Operative Dentistry, Londrina, PR, Brazil.

(c) Universidade de São Paulo - USP, Bauru Dental School, Department of Operative Dentistry, Endodontics and Dental Materials, Bauru, SP, Brazil

Declaration of Interest: The authors certify that they have no commercial or associative interest that represents a conflict of interest in connection with the manuscript.

\section{Corresponding Author:}

Paula Vanessa Pedron Oltramari

Email: pvoltramari@hotmail.com

https://doi.org/10.1590/1807-3107bor-2018.vol32.0047

Submitted: December 18, 2017

Accepted for publication: March 03, 2018

Last revision: August 13, 2018
Abstract: The objective of this study was to assess the effect of a UV lightbased auxiliary illumination on adhesive remnant (AR) removal after orthodontic debonding. Sixty human molars were divided according to the adhesive used for bonding: O-opaque; LF-low fluorescence; and HFhigh fluorescence. After debonding, the teeth were subdivided according to the AR removal method: No UV light or With UV light. After AR removal, the teeth were polished. Direct visual analysis, scanning electron microscopy (SEM) and time quantification for AR removal analyses were performed (Fisher-Freeman-Halton, Fisher's exact, chisquare trend, ANOVA, and independent t-tests; $\alpha=5 \%$ ). Concerning the adhesives, there was no significant difference among direct visual, SEM and time analyses for AR removal ( $p \geq 0.05)$. Regarding AR removal methods, a similarity among the subgroups was verified for direct visual and SEM analyses $(p \geq 0.05)$. However, a significant trend was verified for the with UV light method to produce greater marks, and the no UV light method, to produce a greater rate of samples with AR before polishing $(p=0.015)$. AR removal with light was significantly quicker in comparison with the no UV light method ( $p<0.0001)$. The use of UV light may aid orthodontists in removing AR more thoroughly and in less time. However, they should receive special training to apply this technology, and should never dismiss the final polishing procedure.

Keywords: Orthodontics; Dental Enamel; Fluorescence.

\section{Introduction}

Composite resins are the material of choice for direct bonding of orthodontic accessories, because they present the highest values of bond strength to enamel. ${ }^{1}$ Although direct bonding is a well-established procedure, there remains the challenge of removing adhesive remnants (AR) after debonding of accessories, striving to prevent potential lesions to the enamel surface. ${ }^{2}$ Other difficulties in this stage have been encountered in molars, including reduced accessibility and visualization, as well as the likelihood of having to remove a greater amount of remnant material, since the buccal surface has a larger conditioning area and requires a greater amount of material for accessory bonding. ${ }^{3}$

All the techniques for removing AR after debonding of orthodontic accessories are known to produce different degrees of polishing, and to 
introduce some type of abrasion along with several degrees of enamel loss. To this end, the search for an efficient and safe method for removing AR after debonding has resulted in the introduction of a wide variety of instruments and procedures. ${ }^{4}$

However, the controversy regarding the method that produces fewer changes on the enamel surface in less clinical time remains. On one hand, there is concern about preserving the enamel structure; ${ }_{;}^{4}$ on the other hand, there is the major question of removal time and cost of the material used. ${ }^{5}$ Generally, the methods that offer greater preservation of dental structure demand more clinical time and higher operating costs, eventually causing orthodontists not to adopt them. ${ }^{5}$

In this context, the proposal to remove AR with a UV light system is an interesting alternative that would make it easier to distinguish the difference between the adhesive material and the enamel surface, thus contributing to the preservation of dental structure, and representing a major advance in the orthodontic finishing step. ${ }^{6,7}$

Therefore, this study aimed at assessing the effect of UV light in removing AR after the debonding of orthodontic accessories, taking into account assessment of the enamel surface by direct visual and scanning electron microscopy (SEM) analyses, as well as the amount of time required for the procedure. Accordingly, the following null hypothesis was tested: there was no difference between removing AR after the debonding of orthodontic accessories with or without the aid of UV light.

\section{Methodology}

\section{Sample selection}

The protocol of this study was previously approved by the Ethics Committee of the University of North Paraná.

Ninety human molars indicated for extraction were selected and stored for 7 days in a container with $0.5 \%$ neutral chloramine-T solution for disinfection. Afterwards, the teeth were cleaned and stored at a temperature of about $5^{\circ} \mathrm{C}$, and submerged in distilled water free from any chemical agent, to avoid any interference from the bonding mechanism until the start of the study.
The inclusion criteria included absence of visible defects and damage caused by the extraction process, fissures on the enamel, white lesions on the enamel, caries and restorations on the surface of buccal enamel, exposure to chemical products, and complete rhizogenesis. ${ }^{5}$

\section{Sample preparation, group division and accessory bonding}

Sample size was determined in a pilot study. A power analysis indicated that 10 teeth per group would result in an $80 \%$ chance of obtaining significance at the 0.05 level.

The samples were embedded vertically in PVC tubes with self-polymerizing resin, leaving the crown exposed. ${ }^{8}$ Before bonding the accessories, the teeth were impressed with silicone Express XT $(3 \mathrm{M} \mathrm{ESPE})^{2}$ and the molds were cast after 24 hours with EphoThinTM ${ }^{\mathrm{TM}}$ epoxy resin (Buehler Ltd, Lake Bluff, IL, USA), to record the initial characteristics of the enamel.

Thus, the teeth received orthodontic tubes (ref HI0 10081787, Roth Prescription, 3M), bonded by one single calibrated operator, with the following adhesive systems: $O(n=20)$, Enlight (Ormco/Corporation Glendora, USA), opaque adhesive; LF ( $n=20)$, Transbond Color Change (3M/Unitek Corporation, Monrovia, USA), low fluorescence; and HF ( $\mathrm{n}=20)$, Orthocem UV Trace (FGM, City, Brazil), high fluorescence.

The adhesive systems were used according to the manufacturer's instructions. Bonding was performed by carefully adjusting the tubes in the center of the clinical crowns of the teeth. ${ }^{9}$ The adhesive systems were polymerized with an SDI Radii Cal light-curing device (Dental Limited, Bayswater, VIC, Australia).

The adhesive systems chosen for the present study were selected among the systems available on the market, recommended for orthodontic accessory bonding, and requiring some level of fluorescence when sensitized by ultraviolet light, or, in the absence of fluorescence, high contrast with the enamel (opacity). This would allow the adhesive system to be distinguished from the enamel surface.

After bonding, the samples were stored in distilled water at $37^{\circ} \mathrm{C}$ for 24 hours..$^{10}$ After this time, the shear bond strength test was performed on a universal 
testing machine (DL 2000 EMIC) until the accessories were removed, in order to standardize debonding of the orthodontic accessories. Afterwards, the buccal surfaces of the samples were assessed with a 10X stereoscopic magnifier (BVM100, Bel Photonics, Bel Microimage Analyser, Monza, Italy) to determine the Adhesive Remnant Index (ARI), conducted by two examiners not involved in the bonding process, but previously calibrated (kappa $>0.8$ ) in a blind analysis. ${ }^{11}$ The amount of AR to be removed by the techniques tested in the present study was thus standardized by selecting 60 teeth presenting ARI 1 (less than half of the material bonded to the tooth). ${ }^{11}$ Some teeth were excluded at this time to allow the same amount of AR remnant to be removed, thus providing standardization of the removal technique. These samples were randomly divided into 3 groups according to the adhesive system used ( $\mathrm{O}, \mathrm{LF}$, and $\mathrm{HF})$.

Each group was randomly subdivided into 2 subgroups $(\mathrm{n}=10)$, according to the method for removing AR: No light (NL), multi-bladed 30-blade tungsten carbide bur FF 9714 (JET, Beavers Dental Products Ltd., Morrisburg, Canada), coupled to a high speed pen (Cobra LED Ultra Vision ${ }^{\mathrm{TM}}$, Gnatus) with refrigeration (water) and reflector light (Figure 1a); or With light (WL), multi-bladed 30-blade tungsten carbide bur FF 9714 (JET, Beavers Dental Products Ltd., Morrisburg, Canada), coupled to a high speed pen with refrigeration (water) and a UV light auxiliary illumination system (Cobra LED Ultra Vision ${ }^{\mathrm{TM}}$, Gnatus) (Figure 1b).

This auxiliary illumination system has an LED coupled to the tip of the headpiece (high speed), which emits UV light and reveals adhesive fluorescence in contrast with the enamel.

The burs used for removal were replaced after every five samples for all the subgroups. ${ }^{8}$ The same operator removed the adhesive remnants in all the groups.

\section{Time analysis}

The time required for removing the AR from each sample was quantified with the aid of a second operator. The time-keeping began after the bur was placed on the dental surface having the AR, and ended after total removal of the AR, verified by means of visual inspection, simulating a clinical situation..$^{5,12}$

\section{Direct visual analysis}

After removing the AR, the specimens were coded to allow blind direct visual analysis, performed by two specialists (restorative dentistry and orthodontics) not involved in the study. This analysis was carried out by observing the dental surface under a dental reflector light and by the naked eye, in order to simulate routine clinical conditions as closely as possible. The following criteria were used to make this assessment: $a$. Absence of removal marks; $b$. Presence of soft removal marks; c. Presence of more evident removal marks; and d. Presence of AR. Examiner calibrations were performed by repeatedly assessing the samples. After calibration, the examiners performed two individual assessments at a 15-day interval, achieving kappa $>0.8$. Then, the examiners performed a joint analysis of the samples, the results of which were obtained by consensus.
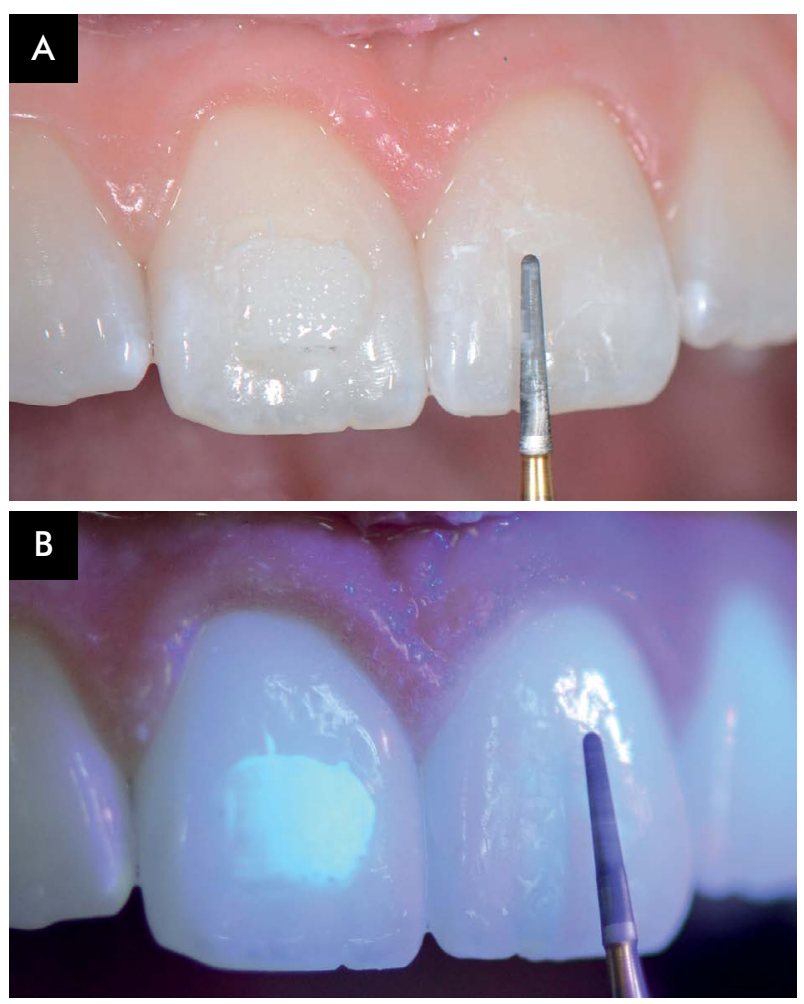

Figure 1. Methods tested for the removal of AR: a) No light (NL), multi-bladed 30-blade tungsten carbide bur coupled to a high-speed pen and reflector light; b) With light (WL), multibladed 30-blade tungsten carbide bur coupled to a high-speed pen using the UV light auxiliary illumination system. 
After making the direct visual assessment, the teeth were again impressed with silicone; 24 hours later, the molds were cast with epoxy resin (Buehler) to allow SEM analysis of the replicates at a later time.

Next, the samples of the NL and WL subgroups were polished with fine-grained aluminum oxide disc (Sof-Lex, 3M ESPE, St Paul, USA) for 30 seconds, felt disc, and Enamelize aluminum oxide paste (Cosmedent, Chicago, USA) for 30 seconds at low speed. After completing this step, all the subgroup samples were again impressed with silicone, and were duplicated in epoxy resin after 24 hours for SEM analysis (after polishing) at a later time.

\section{Scanning electron microscopy (SEM) analysis}

SEM assessment ${ }^{8}$ was performed by randomly selecting $40 \%$ of the replicates from each subgroup $(\mathrm{NL}, \mathrm{n}=4 ; \mathrm{WL}, \mathrm{n}=4)$, in the three steps of the study (initial, after removal of $\mathrm{AR}$, and after polishing).

SEM analysis was performed by inserting the images in a Power Point file and coding them to allow blind analysis of the results. Two previously calibrated (kappa > 0.8) specialists (restorative dentistry and orthodontics) performed this analysis by consensus. The same parameters used in the clinical analysis were considered in comparing the images obtained at different experimental periods (initial, after removal of $\mathrm{AR}$, and after polishing).

\section{Statistical analysis}

Statistical analysis was performed using the Statistical Package for Social Sciences version 20.0 and BioStat software, with a 95\% confidence interval and 5\% significance level. The Fisher-Freeman-Halton test, Fisher's exact test, and chi-square trend test were used (direct visual and SEM assessments), as well as ANOVA and independent t-test (for determining AR removal time).

\section{Results}

Figures 2 and 3 show the enamel aspect after removal of the adhesive remnants (AR), and explain the criteria used to assess the samples by direct visual (Figure 2) and SEM (Figure 3) analyses, where the following marks or absence thereof are observed: a. intact dental enamel, absence of removal marks; b. dental enamel with soft removal marks; c. dental enamel with more evident removal marks; and d) dental enamel with adhesive remnants after removal.

The results obtained by direct visual analysis, after AR removal and after polishing (Table 1), show no significant difference among the different adhesive systems used $(\mathrm{O}$, $\mathrm{LF}$, and HF; $\mathrm{p}=.07$ and $\mathrm{p}=.41$, respectively) or between the different AR removal methods (NL and WL; $p=.05$ and $p=.151$, respectively). However, a threshold value was verified in the comparison between the removal methods (NL and WL) using direct visual assessment after AR removal $(p=.05)$. Hence, the chi-square trend test was performed and indicated a significant trend $(\mathrm{p}=.0150)$ toward a higher rate of marks produced by the WL method in comparison with the NL method, and a higher rate of samples with AR produced by the NL method (Table 1).

It was also verified that the removal of AR was significantly quicker in the WL group, in comparison with the NL group $(p<.0001)$ (Table 3$)$.

\section{Discussion}

This study assessed the effect of using an ultraviolet (UV) light-based auxiliary illumination system to remove AR after debonding of orthodontic accessories, taking into account the enamel surface aspect (direct visual and SEM analyses) and the clinical time required for the procedure.

A method for removing AR after the debonding of accessories, yet allowing the enamel structure to be preserved, still has to be defined, and poses a challenge to orthodontists ${ }^{4,13}$. The present study showed that statistically similar aspects were obtained on the enamel surfaces after removal of AR, verified both by direct visual (Table 1) and SEM (Table 2) analyses, according to a comparison of the different adhesive systems used $(\mathrm{O}, \mathrm{LF}$, and $\mathrm{HF})$ and removal methods (NL and WL). However, a significant trend was observed toward a higher rate of marks produced by the WL method, in comparison with the NL method (Table 1). A possible explanation for the better performance of the NL method at this stage, in comparison with the WL method, could be attributed to the greater ease of $A R$ visualization in group $W L$, 
which could encourage the professional to completely remove the AR. In this context, a previous study ${ }^{14}$ showed that in vitro enamel loss is higher than the actual clinical loss, since the possibility of better visualization of the remnant adhesive system induces greater removal, thus compromising the enamel surface. Thus, although AR may be clinically easier to visualize by UV light, it is worth emphasizing that care should be taken not to be overeager to remove $i^{13}$. Another consideration worth mentioning is the importance of professional training in applying new technologies that produce benefits for the patient. ${ }^{15}$

On the other hand, it was found that the NL method produced a higher rate of samples with AR. In addition to the issue of excessive removal of dental structure when removing $A R$, another factor of concern to professionals is that AR may increase bacterial plaque accumulation, thus increasing the risk of carious lesions and esthetic-compromising changes in enamel color. ${ }^{16}$ Most patients who finish orthodontic treatment may find that some amount of AR still remains on their teeth, because it is difficult to completely remove all the residual composite, though the surface may clinically seem to be clean. ${ }^{17}$ When the color of the adhesive system is similar to the natural tooth color, a careful examination with a manual instrument, such as a dental explorer, should be performed after adhesive removal and polishing of tooth surfaces, in order to verify the color more accurately. ${ }^{18}$

It should be noted that after polishing, both direct visual (Table 1) and SEM (Table 2) analysis results showed similarities between the removal methods (NL and WL), with a significant increase in the number of samples with no removal marks, or else soft removal marks, thus indicating that the rate of surfaces with evident marks was reduced. This emphasizes the importance of polishing, especially after removal of AR by multi-bladed tips at high speed. That is because this step enables obtaining a smooth and shiny surface. ${ }^{4,19}$
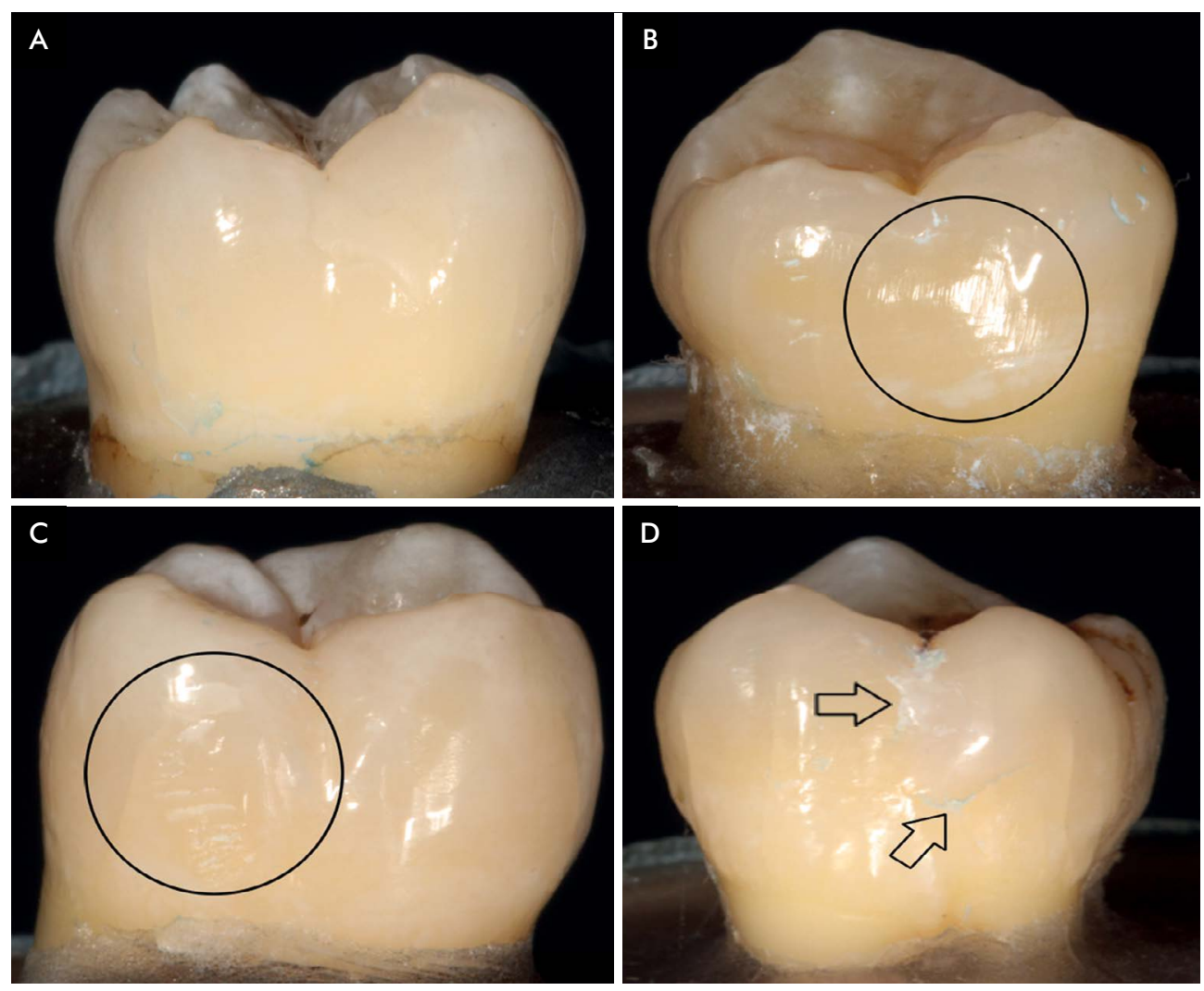

Figure 2. Criteria for direct visual analysis to evaluate the appearance of enamel after removing adhesive remnants: a) intact dental enamel, absence of removal marks; b) dental enamel with the presence of soft removal marks; c) dental enamel with more evident removal marks; d) dental enamel with the presence of adhesive remnants after removal ( $)$ ). 

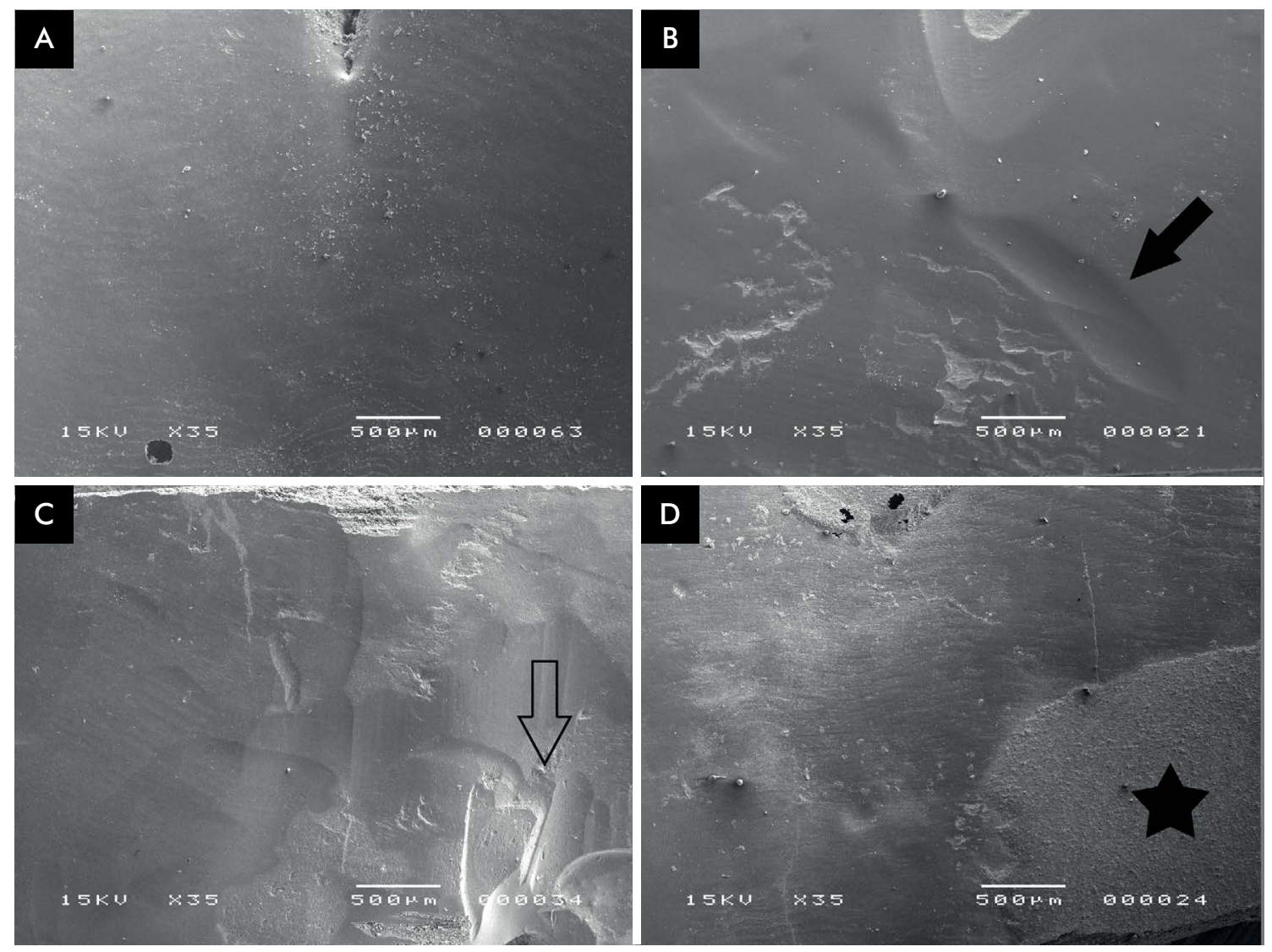

Figure 3. Criteria for scanning electron microscopy analysis to evaluate the appearance of enamel after removing adhesive remnants (35X magnification): a) intact dental enamel, absence of removal marks; b) dental enamel with the presence of soft removal marks (í); c) dental enamel with more evident removal marks (仓); d) dental enamel with the presence of adhesive remnants after removal ( $\star$ ).

Table 1. Direct visual analysis after the removal of adhesive remnants (AR) and after polishing, considering the different systems tested (O: Opaque; LF: low fluorescence; HF: high fluorescence) and the methods for removing AR (NL: no UV light; WL: With UV light).

\begin{tabular}{|c|c|c|c|c|c|c|c|c|c|c|}
\hline \multirow{4}{*}{ Score } & \multicolumn{5}{|c|}{ Direct visual analysis after AR removal } & \multicolumn{5}{|c|}{ Direct visual analysis after polishing } \\
\hline & \multicolumn{3}{|c|}{ Type of resin } & \multicolumn{2}{|c|}{ Removal method } & \multicolumn{3}{|c|}{ Type of resin } & \multicolumn{2}{|c|}{ Removal method } \\
\hline & O & LF & $\mathrm{HF}$ & $\mathrm{NL}$ & $\mathrm{WL}$ & $\mathrm{O}$ & LF & $\mathrm{HF}$ & $\mathrm{NL}$ & WL \\
\hline & $\mathrm{n}(\%)$ & $\mathrm{n}(\%)$ & $\mathrm{n}(\%)$ & $\mathrm{n}(\%)$ & $n(\%)$ & $\mathrm{n}(\%)$ & $\mathrm{n}(\%)$ & $n(\%)$ & $n(\%)$ & $n(\%)$ \\
\hline Absence of marks & $0(0)$ & $0(0)$ & $0(0)$ & $0(0)$ & $0(0)$ & $4(20)$ & $5(25)$ & $7(35)$ & $11(36.7)$ & $5(16.7)$ \\
\hline Soft marks & $13(65)$ & $8(40)$ & $13(65)$ & 19(63.3) & $15(50)$ & $11(55)$ & $7(35)$ & $9(45)$ & $14(46.7)$ & $13(43.3)$ \\
\hline Evident marks & $5(25)$ & $6(30)$ & $7(35)$ & $5(16.7)$ & 13(43.3) & $5(25)$ & $5(25)$ & $4(20)$ & $4(13.3)$ & 10(33.3) \\
\hline With AR & $2(10)$ & $6(30)$ & $0(0)$ & $6(20)$ & $2(6.7)$ & $O(0)$ & $3(15)$ & $0(0)$ & $1(3.3)$ & $2(6.7)$ \\
\hline Total & $20(100)$ & $20(100)$ & $20(100)$ & $30(100)$ & $30(100)$ & $20(100)$ & $20(100)$ & $20(100)$ & $30(100)$ & $30(100)$ \\
\hline$p$-value & \multicolumn{3}{|c|}{$8.26, p=0.07^{*}$} & $\begin{array}{r}5.86, p \\
5.92, p=\end{array}$ & $\begin{array}{l}=0.05^{* *} \\
0.0150^{* * *}\end{array}$ & \multicolumn{3}{|c|}{$5.98, p=0.41^{*}$} & \multicolumn{2}{|c|}{$5.15, p=0.151^{* *}$} \\
\hline
\end{tabular}

*Fisher-Freeman-Halton test; ${ }^{* *}$ Fisher's exact test; ${ }^{* * *}$ Chi-square trend test, significant at $\mathrm{p}<0.05$.

Considering the SEM analysis after AR removal and after polishing (Table 2), there was no significant difference among the different adhesive systems used $(\mathrm{O}, \mathrm{LF}$, and $\mathrm{HF} ; \mathrm{p}=.06$ and $p=.164$, respectively), or between the different methods for removing $A R(N L$ and $\mathrm{WL} ; \mathrm{p}=.81$ and $p=.47$, respectively).

Another relevant clinical aspect refers to the time spent performing the removal of AR after the debonding of orthodontic accessories. Technological development seeking to decrease the time of dental procedures is justified by the benefits that it may bring to patients and professionals. Regarding the patient, time of dental care will be reduced, offering more comfort, especially when dispensing child and elderly care, and 
Table 2. SEM assessment after the removal of adhesive remnants (AR) and after polishing, considering the different systems tested (O: Opaque; LF: low fluorescence; HF: high fluorescence) and the methods for removing AR (NL: no UV light; WL: With UV light).

\begin{tabular}{|c|c|c|c|c|c|c|c|c|c|c|}
\hline \multirow{4}{*}{ Score } & \multicolumn{5}{|c|}{ SEM analysis after AR removal } & \multicolumn{5}{|c|}{ SEM analysis after polishing } \\
\hline & \multicolumn{3}{|c|}{ Type of resin } & \multicolumn{2}{|c|}{ Removal method } & \multicolumn{3}{|c|}{ Type of resin } & \multicolumn{2}{|c|}{ Removal method } \\
\hline & O & LF & $\mathrm{HF}$ & $\mathrm{NL}$ & WL & 0 & LF & $\mathrm{HF}$ & $\mathrm{NL}$ & WL \\
\hline & $n(\%)$ & $n(\%)$ & $n(\%)$ & $n(\%)$ & $n(\%)$ & $n(\%)$ & $\mathrm{n}(\%)$ & $n(\%)$ & $n(\%)$ & $n(\%)$ \\
\hline Absence of marks & $0(0)$ & $2(25)$ & $0(0)$ & $1(36.7)$ & $1(16.7)$ & $0(0)$ & $2(25)$ & $4(0)$ & $3(25)$ & $3(25)$ \\
\hline Soft marks & $3(37.5)$ & $0(0)$ & $2(25)$ & $2(46.7)$ & $3(43.3)$ & $4(50)$ & $2(25)$ & $3(25)$ & $6(50)$ & $3(25)$ \\
\hline Evident marks & $3(37.5)$ & $6(75)$ & $6(75)$ & $7(13.3)$ & 8(33.3) & $3(37.5)$ & $1(75)$ & $1(75)$ & $1(8.3)$ & $4(33.3)$ \\
\hline With AR & $2(25)$ & $0(0)$ & $0(0)$ & $2(3.3)$ & $0(6.7)$ & $1(12.5)$ & $3(0)$ & $O(0)$ & $2(16.7)$ & $2(16.7)$ \\
\hline Total & $8(100)$ & $8(100)$ & $8(100)$ & $12(100)$ & $12(100)$ & $8(100)$ & $8(100)$ & $8(100)$ & $12(100)$ & $12(100)$ \\
\hline $\mathrm{p}$-value & \multicolumn{3}{|c|}{$9.24, p=0.06^{*}$} & \multicolumn{2}{|c|}{$2.21, p=0.81^{* *}$} & \multicolumn{3}{|c|}{$8.73, p=0.164^{*}$} & \multicolumn{2}{|c|}{$2.80, p=0.47^{* *}$} \\
\hline
\end{tabular}

*Fisher-Freeman-Halton test; ${ }^{* *}$ Fisher's exact test.

Table 3. Time (in seconds) for removal of adhesive remnants $(A R)$, considering the different systems tested (O: Opaque; LF: low fluorescence; HF: high fluorescence) and the methods for removing AR (NL: no UV light; WL: With UV light).

\begin{tabular}{ccccc}
\hline \multicolumn{4}{c}{ AR Removal time - Mean (SD) } \\
\hline \multicolumn{4}{c}{ Type of resin } \\
\hline O & LF & HF & NL & WL \\
$16.4(6.8)$ & $16.5(6.2)$ & $14.3(3.4)$ & $18.6(6.1)$ & $12.8(3.2)$ \\
& $p=0.5873^{*}$ & & $p<0.0001^{* *}$ \\
\hline *ANOVA; $^{* *}$ Independent t-test, Significant.
\end{tabular}

for patients with temporomandibular dysfunction, who find it difficult to remain long periods with their mouth open. Moreover, the professional may also be benefited, since his efficiency will be considered greater when determining cost effectiveness. ${ }^{5}$ The present study verified that using a UV light auxiliary system (WL) allowed a significantly quicker removal of AR (Table 3) relative to method NL. The examiner reported greater ease of removal from a clinical standpoint; that is because use of UV light allowed observing the difference in fluorescence between AR and the dental structure more clearly, regardless of the adhesive system tested $(\mathrm{O}, \mathrm{LF}$, and HF) (Table 3). Another study has already shown that removal of AR was statistically quicker when performed with multibladed tips at high speed; ${ }^{19}$ however, there are no previous scientific studies in orthodontics associating AR removal time with UV light. Thus, the results of the present study showed that the presence of UV light accelerates the procedure, and promotes a clinical time gain with greater technical agility and service efficiency, as well as reducing costs.

The authors suggest that, for more efficient removal of orthodontic accessories, UV light may be used in association with multi-bladed burs at high speed until visualizing a thin layer of $A R$, and then completing the removal with specific finishing and polishing tips at low speed. This method applied to clinical practice would allow a fast, complete, and safe removal of $A R$, while also preserving the enamel surface of the patient.

\section{Conclusion}

The use of UV light (WL) for removing AR after debonding of orthodontic accessories produced results similar to the non-UV (NL) light removal technique on the enamel surface, but in less clinical time. However, there was a significant clinical trend toward a higher rate of marks produced by the WL method in comparison with the NL method, which indicates that professional training is essential to obtain the best results.

\section{References}

1. Parrish BC, Katona TR, Isikbay SC, Stewart KT, Kula KS. The effects of application time of a self-etching primer and debonding methods on bracket bond strength. Angle Orthod. 2012 Jan;82(1):131-6. https://doi.org/10.2319/020411-82.1

2. Pignatta LM, Duarte Junior S, Santos EC. Evaluation of enamel surface after bracket debonding and polishing. Dental Press J Orthod. 2012;17(4):77-84. https://doi.org/10.1590/S2176-94512012000400017 
3. Murray PG, Millett DT, Cronin M. Bonded molar tubes: a survey of their use by specialist orthodontists. J Orthod. 2012 Jun;39(2):129-35. https://doi.org/10.1179/1465312512Z.00000000016

4. Ulusoy $C$. Comparison of finishing and polishing systems for residual resin removal after debonding. J Appl Oral Sci. 2009 May-Jun;17(3):209-15. https://doi.org/10.1590/S1678-77572009000300015

5. Karan S, Kircelli BH, Tasdelen B. Enamel surface roughness after debonding. Angle Orthod. 2010 Nov;80(6):1081-8. https://doi.org/10.2319/012610-55.1

6. Hamba Y, Yamagata S, Akasaka T, Uo M, lida J, Watari F. Preparation and properties of fluorescent orthodontic adhesives containing Y2O3:Eu3 particles. Nano Biomedicine. 2013;5(2):75-84.

7. Yamagata S, Iwasaki $H$, Hamba Y, Nakanishi K, Ushijima $\mathrm{N}$, Abe $\mathrm{S}$ et al. An evaluation of fluorescent orthodontic adhesives contining Eu-doped $\mathrm{ZnO}$ at room temperature. Nano Biomedicine. 2013;5(1):31-8.

8. Faria-Júnior ÉM, Guiraldo RD, Berger SB, Correr AB, Correr-Sobrinho L, Contreras EF et al. In-vivo evaluation of the surface roughness and morphology of enamel after bracket removal and polishing by different techniques. Am J Orthod Dentofacial Orthop. 2015 Mar;147(3):324-9. https://doi.org/10.1016/j.ajodo.2014.10.033

9. Ireland AJ, Hosein I, Sherriff M. Enamel loss at bond-up, debond and clean-up following the use of a conventional light-cured composite and a resin-modified glass polyalkenoate cement. Eur J Orthod. 2005 Aug;27(4):413-9. https://doi.org/10.1093/ejo/cji031

10. Johnston CD, Burden DJ, Hussey DL, Mitchell CA. Bonding to molars: the effect of etch time (an in vitro study). Eur J Orthod. 1998 Apr;20(2):195-9. https://doi.org/10.1093/ejo/20.2.195
11. Artun J, Bergland S. Clinical trials with crystal growth conditioning as an alternative to acid-etch enamel pretreatment. Am J Orthod. 1984 Apr;85(4):333-40. https://doi.org/10.1016/0002-9416(84)90190-8

12. Eminkahyagil N, Arman A, Cetinşahin A, Karabulut E. Effect of resin-removal methods on enamel and shear bond strength of rebonded brackets. Angle Orthod. 2006 Mar;76(2):314-21. https://doi.org/10.1043/0003-3219(2006)076[0314:EORM $\mathrm{OE}] 2.0 . \mathrm{CO} ; 2$

13. Campbell PM. Enamel surfaces after orthodontic bracket debonding. Angle Orthod. 1995;65(2):103-10.

14. Brown CR, Way DC. Enamel loss during orthodontic bonding and subsequent loss during removal of filled and unfilled adhesives. Am J Orthod. 1978 Dec;74(6):663-71. https://doi.org/10.1016/0002-9416(78)90005-2

15. Brownstein SA, Murad A, Hunt RJ. Implementation of new technologies in U.S. dental school curricula. J Dent Educ. 2015 Mar;79(3):259-64.

16. Chen CS, Hsu ML, Chang KD, Kuang SH, Chen PT, Gung YW. Failure analysis: enamel fracture after debonding orthodontic brackets. Angle Orthod. 2008 Nov;78(6):1071-7. https://doi.org/10.2319/091907-449.1

17. Gwinnett AJ, Gorelick L. Microscopic evaluation of enamel after debonding: clinical application. Am J Orthod. 1977 Jun;71(6):651-65. https://doi.org/10.1016/0002-9416(77)90281-0

18. Namura Y, Tsuruoka T, Ryu C, Kaketani M, Shimizu N. Usefulness of orthodontic adhesive-containing fluorescent dye. Eur J Orthod. 2010 Dec;32(6):620-6. https://doi.org/10.1093/ejo/cjq015

19. Zarrinnia K, Eid NM, Kehoe MJ. The effect of different debonding techniques on the enamel surface: an in vitro qualitative study. Am J Orthod Dentofacial Orthop. 1995 Sep;108(3):284-93. https://doi.org/10.1016/S0889-5406(95)70023-4 